Portland State University

PDXScholar

Environmental Science and Management

Faculty Publications and Presentations

Winter 2012

\title{
The Effects of Scenario Planning on Participant Perceptions of Learning Organization Characteristics
}

\author{
Melissa Haeffner \\ Portland State University \\ Deanna Leone \\ Colorado State University \\ Laura Coons \\ Colorado State University \\ Thomas Chermack \\ Colorado State University
}

Follow this and additional works at: https://pdxscholar.library.pdx.edu/esm_fac

Part of the Organizational Behavior and Theory Commons

Let us know how access to this document benefits you.

\section{Citation Details}

Haeffner, M, D Leone, L Coons, and T Chermack (2012) "The effects of scenario planning on participant perceptions of learning organization characteristics." Human Resource Development Quarterly, 23(4), 519-542. DOI: $10.1002 /$ hrdq. 21147

This Article is brought to you for free and open access. It has been accepted for inclusion in Environmental Science and Management Faculty Publications and Presentations by an authorized administrator of PDXScholar. Please contact us if we can make this document more accessible: pdxscholar@pdx.edu. 


\title{
The Effects of Scenario Planning on Participant Perceptions of Learning Organization Characteristics
}

\author{
Melissa Haeffner, Deanna Leone, Laura Coons, \\ Thomas Chermack
}

\begin{abstract}
Scenario planning is often used by organizations to think about future uncertainties. However, what it does in terms of changing perceptions is difficult to assess and quantify. To address this need, this article builds on previous studies documenting the effectiveness of scenario planning. Specifically, this article contributes to the data on perceptions of learning organization characteristics. This study compiles quantitative data gathered from 133 individuals working in 10 United States companies who participated in scenario planning. The Dimensions of the Learning Organization Questionnaire (DLOQ) instrument was used in a pre- and post-test research design and differences were analyzed using a t-test. Results show a positive relationship between scenario planning and improved perceptions of learning organization characteristics for six of the seven dimensions of the DLOQ. Interpretation of the results suggests that scenario planning can change participants' views of their organizations' adaptive learning characteristics.
\end{abstract}

As more information becomes available about what could happen in the future, organizations have devised tools in order to organize, disseminate, and assess this information. One such method is scenario planning. We focus on the particular form of scenario planning that involves stakeholders brainstorming their shared visions in an effort to establish adaptive capacity for future uncertainties. It is positioned as a managerial device to address the limitations of more traditional strategic planning methods that are criticized for being prescriptive and reactive. Meanwhile, scenario planning is said to embrace more emergent and proactive views (Mintzberg, Ahlstrand, \& Lampel, 2005; 
Maruyama, 2004; Kilduff \& Mehra, 1997). Scenario planning is used by an increasing variety of organizations to organize for uncertain futures in the spheres of product innovation, nation building, infrastructure development, and climate change. However, it is more than just a tool to help organizations. Studies have demonstrated that scenario planning has benefits for those who participate as well; there appears to be a strong link between the scenario planning method and stakeholder satisfaction (Rowden \& Conine, 2005; Yang, Watkins, \& Marsick, 2004).

Wright, van der Heijden, Burt, Bradfield, and Cairns (2008) acknowledged the virtues of scenario planning, but cautioned that scenario planning experts could fall into the same trap as their predecessors in strategic planning by taking vague and ill-substantiated claims as evidence of competence and effectiveness. In other words, while scenario planning has great promise as a technique that builds uncertainty into the planning process, rigorous research must demonstrate the specific benefits of scenario planning. While data-driven studies are increasingly emerging, they remain somewhat limited by potential sampling errors (Chermack, Lynham \& van der Merwe, 2006; Chermack, van der Merwe, \& Lynham, 2007). For example, some experiments use college students in simulated scenarios instead of employees in businesses facing actual challenges. Although students are readily available and low cost to recruit into a research study, they may introduce a bias, if they have an external motivation to participate (a grade, for instance). Further, it is possible that the scenario experience would differ significantly from participant to participant because the students come from a variety of backgrounds and lack a common current issue or decision context.

Indeed, Bradfield (2008) reported that his students were heavily influenced by the media trends, a crutch that might only affect those who do not have intimate, day-to-day experience with the phenomena. Another sampling error is simply not being able to test a large enough sample. Testing employees takes time away from work, and managers are hesitant to approve of such experiments. For example, a previous study on scenarios and the connection to learning organizational characteristics involved 10 participants (Chermack, Lynham, \& van der Merwe, 2006). Part of the issue with such a small sample size is representative of one of the core challenges in modern organizational research-it can be difficult to recruit voluntary participants in an economic climate that has never before been so lean. The study reported significant differences on participant responses after the scenario planning intervention. Although a $t$-test is a relatively robust test that can be an effective analysis tool with small sample sizes, a larger and more diverse sample would increase the generalizability of the findings.

In an attempt to address the sample size issue in the prior evaluations of the effectiveness of scenario planning, the present study compiles data gathered from 133 individuals working in 10 U.S. companies who participated in 
scenario planning in the last quarter of 2010. Much of the scenario planning research consists of qualitative case studies that establish nuances about the process, but few studies attempt to establish general relationships through multi-source, data-driven research. Thus, the research presented here is a unique contribution. It replicates and advances the task of a previous research study, boasts one of the largest sample sizes in scenario planning research to date, and features data collected from 10 different industries.

\section{Purpose of the Article, Problem, and Research Question}

This article has two general purposes: (1) to present a replication study with a much larger sample size than the previous study, and (2) to contribute to the broader research base of scenario planning for the purposes of organizational learning through a large and diverse sample (as compared to existing studies on scenario planning). Both of these purposes address a central problem in scenario planning, which is a lack of rigorous research (Bradfield, Wright, Burt, Cairns, \& van der Heijden, 2005).

The focus of this study is the relationship between scenario planning and perceptions of learning organization characteristics. In scenario planning literature, there is a general consensus that scenario planning promotes a learning orientation in organizations (De Geus, 1988; Michael, 1995; van der Heijden, 1997, 2005; Schwartz, 1996) and that scenario planning can be a tool for organizations intent on becoming learning organizations (Chermack, 2011; De Geus, 1988). This study seeks to assess the quality of these claims using a multi-source data sample.

The more specific purpose of this study is:

To determine if there is a relationship between scenario planning and participant perceptions of learning organization characteristics.

The majority of the scenario planning literature takes a learning approach to strategy rather than a financial one. In other words, making sound strategic decisions is based on learning about factors such as the environment, competitors, and industry trends, and then playing with these variables to create meaningful insights about how the future could play out. This approach is decidedly different from simply hiking revenue expectations for business units across the board or constantly cutting costs to survive budget shortfalls.

Previous research has shown that perceptions of learning organization characteristics are a proxy for organizational performance based on measures of stock market performance (Ellinger, Ellinger, Yang, \& Howton, 2003), and increasing these perceptions of learning organization characteristics can influence the actual performance of the organization. Thus, the research question for this study is: Does scenario planning affect participant perceptions of their firms' learning organization characteristics? 


\section{Theoretical Framework}

The theoretical framework for this study combines theories of learning organizations with the practice of scenario planning. These two bodies of research and practice are abstract, and measurement difficulties have posed problems for both. These disciplines have faced a lack of research in general, and because of their more qualitative nature, both encounter obstacles in terms of locating useful strategies for assessing changes in intended characteristics (Bradfield, Wright, Burt, Cairns, \& van der Heijden, 2005). First, the term "learning organization" is described and clarified. Then, this section provides an overview of scenario planning, with particular attention to it as an intervention to deal with uncertainty. Overall, the theoretical framework presented here establishes the rationale for the study, as well as the major theoretical foundations.

Learning, important to an organization's survival, has increasingly become high priority (Michael, 1995): "For many students of strategy, the holy grail is an organization capable of cumulative learning and constant selfrenewal" (Mintzberg, Ahlstrand, \& Lampel, 2005, p. 214). Valuing organizational members' access to knowledge, respecting their contributions, and promoting their engagement are each increasingly important concepts and reflect a modern approach distinct from older "employee as cog" models (De Geus, 1989; Michael, 1995). As organizations seek to maintain resilience in an expanding world, they look inward to build capacity among individual employees. Organizations following the learning model can be identified as those who: value input from those closest to the product (design, sale, etc.) rather than input from just superiors, learn more from their failures than their successes, make knowledge fluid within the organization so that it can be accessed when and where it is needed, and search for knowledge outside of themselves (Mintzberg, Ahlstrand, \& Lampel, 2005, pp. 214-215). In such an organization, "[w] orkplace learning is part of the knowledge capital of the organization ... important as one way of measuring the value added of the human resource development function" (Marsick \& Watkins, 2003, p. 138). Some have critiqued the learning organization theory developed by Watkins and Marsick (2003) as dated; however, we argue that this was precisely the reason for using it. Their theory and method has a history, with a track record of numerous studies with high reliability and validity scores. While there are more modern learning organization authors and instruments (Garvin, Edmondson, \& Gino, 2008; Hannah \& Lester, 2009), the studies using their approach and instruments are scarce. Thus, the use of Watkins and Marsick's heavily substantiated approach seemed appropriate and useful to our research. Additionally, as a form of face validity, the seven dimensions of the learning organization characteristics appear repeatedly in the scenario literature. Finally, Watkins and Marsick's Dimensions of the Learning Organization Questionnaire (DLOQ) has been shown as a proxy for firm financial 
performance in the rigorous study conducted by Ellinger, Ellinger, Yang, and Howton (2003). These factors all make the DLOQ a logical and rigorous choice for learning organization research.

The overall hypothesis that the learning organization community posits that when decision makers within organizations learn to think differently about uncertainty, their learning becomes the driver of change for the organization, replacing the reactionary responsiveness to the ever-changing organizational environment. Subsequently, scenario planning can be used as a tool to manifest organizational learning and as an intentional method for moving the organization through change.

Learning for learning's sake is not the goal, rather to learn strategically, with intent. Four key steps in strategic learning have been outlined as: (1) reinterpret the situation; (2) apply that reinterpretation to policy, action, and the evaluation of action; (3) implement those policies and actions; and (4) continually revise as the situation evolves (Michael, 1995, p. 46).

Learning organizations believe that wide- and large-scale learning improves the ability to anticipate major changes and increases the likelihood that once they recognize impending events, organizational members will be able to take the necessary actions to minimize negative impacts (Bradfield, Wright, Burt, Cairns, \& van der Heijden, 2005; Chermack, 2011; Durance \& Godet, 2010; Schwartz, 1996; Wack, 1985c). Those actions are the final link in the chain-it is not enough simply to connect strategy and learning. These points on strategic learning exemplify some key practical and philosophical intents of scenario planning. Further, they connect scenario planning with learning organization concepts.

\section{Scenario Planning}

Introduced in the 1960s, scenario planning is an organizational strategy tool originally used to develop weapons technology. It has evolved into a methodology for business planning, community building, and initiating major social change initiatives (Schwartz, 1996). Today, scenario planning is increasingly common in organizations as an approach to long-term planning for volatile and unpredictable futures in constantly changing environments (Bradfield, Wright, Burt, Cairns, \& van der Heijden, 2005; Chermack, 2011; Durance \& Godet, 2010; Schwartz, 1996; Wack, 1985c). Probably most widely known for its use at Dutch Royal/Shell in the 1960s and 1970s (Wack, 1985a, 1985b), scenario planning was used by organizational leaders as a strategic tool for anticipating shifts in the future of the oil industry. Scenario planning continues to generate appeal for its ability to build the element of uncertainty into planning and decision making, and has been used by educational administrators, nonprofit executives, business strategists, and government leaders across the world to bring people together to talk and think differently about their organizations 
(Bradfield, et al., 2005; Durance \& Godet, 2010). Scenario planning aims to allow a space for stakeholders to reflect on the most critical uncertainties within their organization. The process is meant to encourage them to shift their thinking as a means to consider the major implications of critical uncertainties in realistic and plausible ways (Schwartz, 1996; van der Heijden, 1997; 2005).

Because of its intrinsically complex nature, as well as its application in such a wide variety of industries and organizational contexts, a single definition of scenario planning is elusive. Depending on the specific use at hand, the concept can vary in its exact implication. For example, as a strategic tool for organizations, scenario planning has been defined as "an internally consistent view of what the future might turn out to be-not a forecast, but one possible future outcome" (Porter, 1985, p. 63). In another, more broadly social context, it has been defined as ". . inherently a learning process that challenges the comfortable conventional wisdoms of the organization by focusing attention on how the future may be different from the present" (Thomas, 1994, p. 6). Yet a third definition is that scenario planning is a means of "creating stories of equally plausible futures and planning as though any one could move forward" (Tucker, 1999, p. 70). While one precise definition of scenario planning has not generated unified agreement, common benefits of scenario planning for organizations are generally agreed to include the following: (1) emphasis on changed thinking, (2) informed narratives of plausible futures, (3) improved decision making about the future, and (4) enhanced human and organizational learning (Chermack, 2011).

In this research study, the following definition of "Performance-Based Scenario Planning" framed the approach to scenario facilitation. It includes a learning organization culture as an expected outcome: "a discipline of building a set of internally consistent and imagined futures in which decisions about the future can be played out, for the purpose of changing thinking, improving decision-making, fostering human and organization learning, and improving performance" (Chermack, 2011, p. 16). This definition was shared with the participants for the study because it underscores the notion that scenario planning is an active, group-based process that involves taking time to consider a key issue or decision that deeply impacts an organization.

\section{Steps in the Scenario-Planning Process}

Participants are asked to simultaneously consider both the internal and external forces impacting the long-term success of an organization in an uncertain environment (Schwartz, 1996). Scenario planning involves a set of phases that bring together key stakeholders to brainstorm the major issues facing their business agenda, as well as the levels of relative impact and uncertainty of each strategic issue within that agenda. The decision makers then narrow down the options and focus on a few critical uncertainties from which participants create plotlines or stories. The use of story has often been thought of as 
a means by which individuals can relate to and make meaning out of information (Pink, 2005, 2006; Shaw, Brown, \& Bromily, 1998). Crafting narratives around abstract or uncomfortable areas of uncertainty is meant to help participants understand the significance of these elements more clearly (van der Heijden, 2005). Moreover, it is expected that participants will be better able to comprehend the greater context in which these key uncertainties exist by creating shared meaning through the use of story (Boje, 1991; Shaw, Brown, \& Bromily, 1998). Thus, creating descriptive and plausible ideas about the future of the organization serves as a vehicle to bring the plotlines to life for participants. The scenarios are then tested for plausibility before moving into the final stages of implementation and an overall assessment of the entire process. The approach to scenario planning studied and implemented as the intervention in this research study is shown in Figure 1 (Chermack, 2011).

In summary, the theoretical framework suggests that organizations that are more learning oriented are better positioned to manage future change while continuously learning from internal actions and external environments. Scenario planning is an activity proposed to aid in establishing learning organization characteristics through this continuous learning orientation.

\section{Figure 1. The Performance-Based Scenario System}

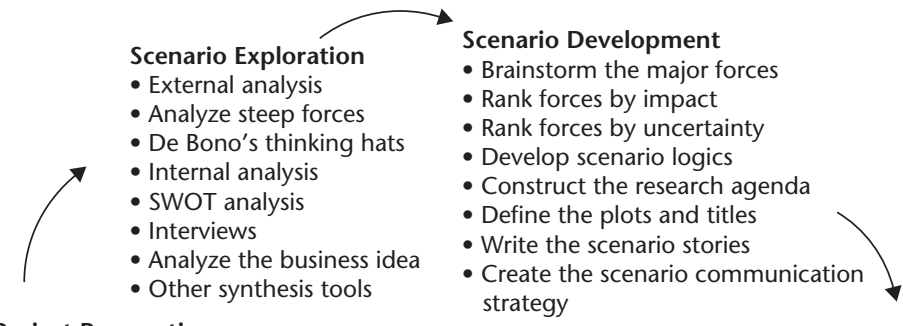

Project Preparation

- Articulate the purpose

- Define the estimated scope and time frame

- Build the scenario team and determine roles

- Articulate the general expected outcomes

- Take measures relative to the expected outcomes

- Construct the project proposal

Inputs

- Stakeholder need

- Problem or issue

- Organization history and culture

- Others

Outputs

- Increased understanding of environmental dynamics

- Ability to see problems or issues in a new way

- Shared understanding of the organization and issues

- Aligned organizational systems

- Robust strategy

- Others
Scenario Implementation

- Wind tunneling

- Examine the initial question

- Scenario immersion

- Test the theory of the business/ business idea

- Analyze current strategies

- Develop signals

- Experiential exercise

- Build resilience and robustness

- Other

Project Assessment

- Revisit purpose

- Take satisfaction measures

- Take knowledge measures

- Take expertise measures

- Take system measures

- Take financial measures

Source: Chermack, 2011 
Hypotheses. While a previous study attempted to explore the relationship between scenario planning and learning organization characteristics (Chermack, Lynham, \& van der Merwe, 2007), the small sample size ( $N=9$ ) was decidedly inadequate to properly assess the relationship. Therefore, the present study is part replication, and part advancement of scenario planning research. Given the theoretical basis and connections described and supported in the theoretical framework, we hypothesize that scenario planning will increase participant perceptions of learning organization characteristics. To specify, we expect that mean scores on all seven constructs of perceptions of the learning organization as measured by the DLOQ will increase as a result of participation in scenario planning. Thus, we hypothesize that results will show a general change in participant perceptions of their organization's ability to learn and adapt. The general null hypothesis and these two hypotheses are shown symbolically as follows:

$$
\begin{aligned}
& \text { H0: } \mu \mathrm{D}=0 \\
& \mathrm{H} 1: \mu \mathrm{D} \geq 0 \\
& \mathrm{H} 2: \Delta \mu \mathrm{D}
\end{aligned}
$$

According to the seven dimensions of the learning organization measured by the DLOQ, the corresponding, more specific seven hypotheses are as follows:

$\mathrm{H1}$ : Individuals who engage in scenario planning will tend to perceive their organization to increase its creation of continuous learning opportunities.

H2: Individuals who engage in scenario planning will tend to perceive their organization to increase its promotion of inquiry and dialogue.

H3: Individuals who engage in scenario planning will tend to perceive their organization to increase its encouragement of collaboration and team learning.

$\mathrm{H} 4$ : Individuals who engage in scenario planning will tend to perceive their organization to increase its empowerment of people toward a collective vision.

H5: Individuals who engage in scenario planning will tend to perceive their organization to increase its ability to connect the organization to its environment.

H6: Individuals who engage in scenario planning will tend to perceive their organization to increase its ability to establish systems to capture and share learning.

H7: Individuals who engage in scenario planning will tend to perceive their organization to increase its ability to provide strategic leadership for learning. 
Method. The purpose of this study is to investigate the effects of scenario planning on participant perceptions of their firm's learning organization characteristics. The following sections describe the sample, instrumentation, data collection, and data analysis strategies.

Sample. This study used a non-random convenience sample composed of participants in 10 scenario planning projects in 10 different organizations. A nonrandom convenience sample was purposeful in order to attract organizations that were willing to participate and try new techniques. Further, scenario exercises are often conducted with groups of 10-30 employees of various ranks. In order to obtain a larger sample size, it is necessary to pool the participants from multiple scenario exercises. While the study lacks random sampling, one benefit from the approach of multi-organization convenience sampling is the representation of multiple industries, organization sizes, and cultures. These organizations were also chosen for their diversity in industry, scope, and scale. Health, educational, and commercial organizations were represented, as were small (20 or fewer employees/volunteers) and large $(10,000$ or more employees) organizations. Each organization had representation from its top management team (in some cases the CEO participated), as enacting change would require decision-making authority.

Survey respondents were chosen on the basis of their participation in a scenario planning process conducted by 10 teams of facilitators. While the facilitators were similarly trained, each team was given creative freedom to customize the workshops according to each organization, context, and industry demands as scenario-planning practices demand. Thus, while there was some variation in the specific project details, all projects followed the same general framework (see Figure 1) and were generally advised by a single project leader who oversaw all 10 scenario projects.

Organizations were chosen based on willingness to participate (authorized by an organizational leader), time constraints (ability to complete fulllength projects within a specific timeline), and space limitations (geographic proximity). Participating organizations represented a diverse set of industries, including health care, technology, education, nonprofits, and others. Data from all projects were aggregated for a total of 133 participants $(N=133)$.

Instrument. The Dimensions of Learning Organization Questionnaire (DLOQ) developed by Watkins and Marsick (1996; 1997) was chosen to measure indicators of perceptions of learning organizations based on previous success (Chermack, Lynham, \& van der Merwe, 2006), as well as documented evidence of meeting validity and reliability (Yang, Watkins, \& Marsick, 2004). Further rationale for using Watkins and Marsick's approach and instrument are its long-term use with high reliability and validity of scores in multiple studies, face validity in terms of how the seven dimensions map to expected scenario planning outcomes, and links to financial performance. In Watkins \& 
Marsick's (1997) words, the DLOQ was "built on the idea that change must occur at every level of learning-from individual to group to organizational to environmental-and that these changes must become new practices and routines that enable and support the ability to use learning to improve performance" (p. 135). The DLOQ is divided into three sections in which the respondent answers a 6-point Likert scale question at the individual level ("In my organization, people openly discuss mistakes in order to learn from them"), at the team level ("In my organization, team/groups focus both on the group's task and on how well the group is working"), and at the organizational level ("My organization uses two-way communication on a regular basis, such as suggestion systems, electronic bulletin boards, or town hall/open meetings").

The DLOQ measures characteristics of learning organizations on seven dimensions: (1) continuous learning, (2) dialogue and inquiry, (3) collaboration and team learning, (4) embedded systems, (5) empowerment, (6) system connections, and (7) leadership. Continuous learning refers to opportunities for growth that are provided through the job, such as ongoing education. ("In my organization, people can get money and other resources to support their learning.") Dialogue and inquiry refer to the extent to which the organization supports employees to express their views whether they are questioning, giving feedback, or experimenting. ("In my organization, people give open and honest feedback to each other.") A learning organization encourages collaboration and team learning by creating work expectations and culture around learning together. ("In my organization, teams/groups revise their thinking as a result of group discussions or information collected.") Embedded systems refer to both high- and low-technology systems that are integrated into work and allow employees to share learning. ("My organization enables people to get needed information at any time quickly and easily.")

Empowerment refers to the perception that employees are involved in setting the agenda, able to take ownership in decision making, and are accountable to the collective vision. ("My organization invites people to contribute to the organization's vision.") System connections refer to the respondent's perception of how well employees see the impact of their contributions, as well as how well the organization incorporates outside views. ("My organization encourages everyone to bring the customers' views into the decisionmaking process.") Lastly, leadership refers to how the respondent feels the organization rewards people who use learning strategically for business results. ("In my organization, leaders generally support requests for learning opportunities and training.") The DLOQ, in essence, evaluates how effective the organization is at providing the resources for individuals to acquire knowledge (1), at providing a safe space for individuals to share knowledge $(2,3,5)$, and at providing avenues for the dissemination of knowledge through individual organization members $(4,6,7)$. 
Prior reports of instrument reliability estimates. Given that the DLOQ has been well used and documented, Table 1 summarizes the reliability estimates from a sample of previous research studies across varying cultures as provided in Song and Chermack (2008).

Prior reports of instrument construct validity. Yang (2003) has led the statistical analyses in a series of studies that establish evidence of the construct validity of scores from the DLOQ (Yang, 2003; Yang, Watkins \& Marsick, 2004; Ellinger, Ellinger, Yang, \& Howton, 2003). Using Confirmatory Factor Analyses (CFA), these studies consistently reported RMSEAs less than .08, suggesting strong evidence for the seven-factor structure of the DLOQ. Interestingly, the fit indices reported by Yang (2003) favored the 21-item version of the DLOQ (which we used in our research) over the original 42-item instrument. Song, Joo, and Chermack (2009) published a summary of fit indices for a sample of measurement models using the DLOQ. The summary is provided again here to show evidence of construct validity from multiple samples, across multiple cultures (Table 2).

Some limitations exist with the instrument; namely, that it is based on self-reporting and it cannot account for time lags. First, the DLOQ is a selfreported perception measure. While the accuracy of self-reporting is always a concern, instruments with long histories of use such as the DLOQ can become proxies for behaviors and, therefore, eventually overcome this limitation through continued use and reporting of results. Second, the DLOQ cannot account for a lag in learning initiatives, especially in surveys conducted on the same day. Learning is a process that can include unlearning as well as steep learning curves that cannot be captured by the instrument. Thus, measurements might actually show setbacks at times. However, the indication of learning as a form of change is still captured.

Data Collection. Data were collected in two phases. The first data collection phase occurred prior to the start of the scenario workshops. Participants were asked to complete the short version (21 items) of the Dimensions of Learning Organization Questionnaire (DLOQ) at a meeting held within each of the 10 organizations to describe the research project, present the IRB consent forms, inform participants that their participation was completely anonymous and voluntary, and that they could withdraw from the study at any time. These meetings were generally held two weeks prior to the start of the scenario-planning facilitation, although this time period was slightly shorter or slightly longer in some cases.

Participants were given approximately one half-hour to complete the DLOQ. They were asked to select a code known only to them and write it on their paper survey so that the facilitators could match their pretests to their posttests. Participants were instructed to place consent forms in a separate folder from the surveys to ensure confidentiality. They were also asked to remember their code, as they would need it again for the posttest. This coding scheme would ensure anonymity while allowing researchers to match 


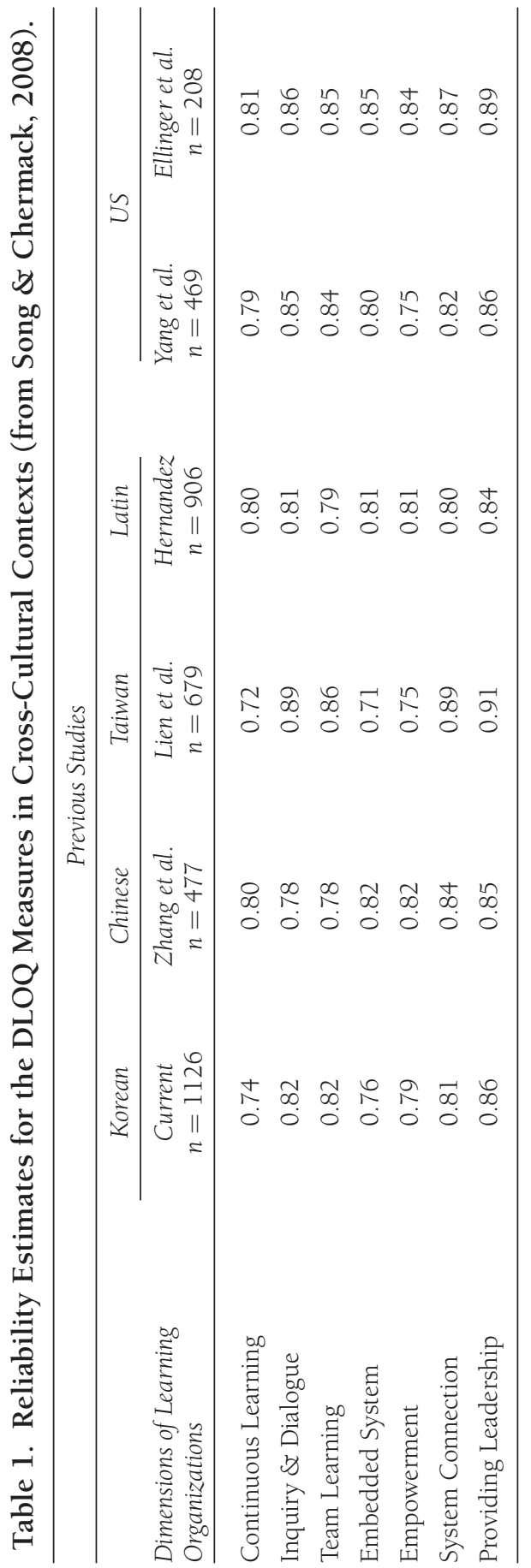




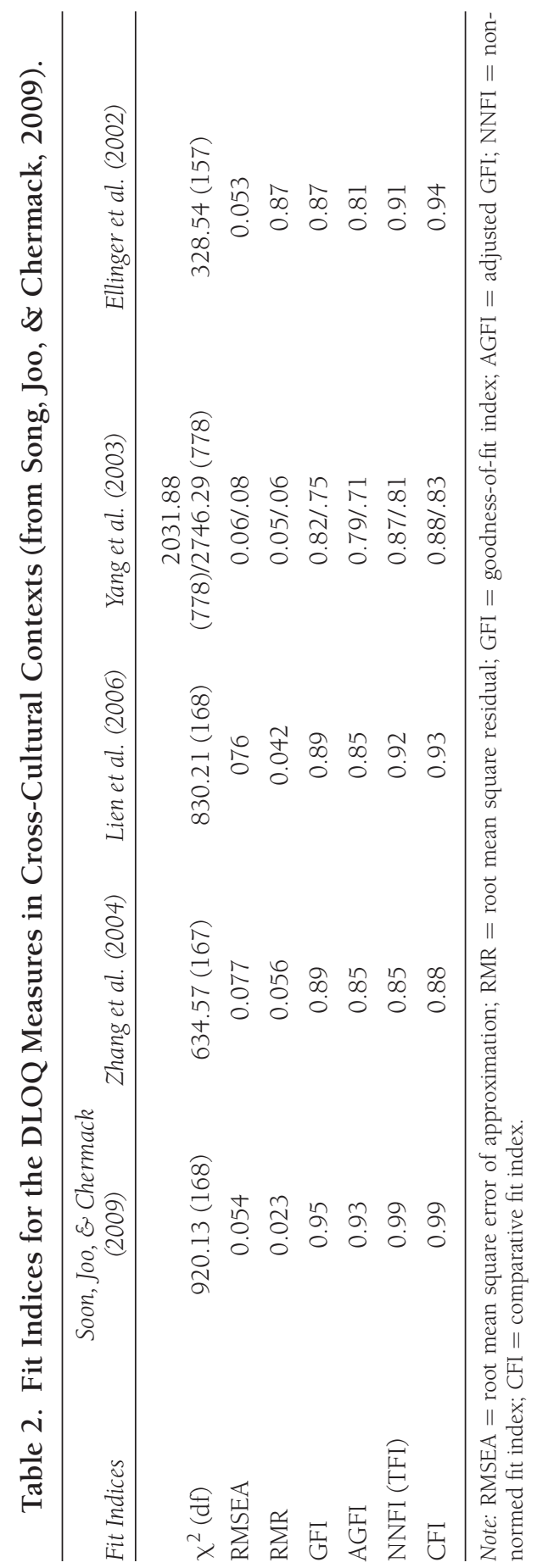


before-and-after responses of the same individual. Files were saved according to strict institutional guidelines, although researchers never had access to the actual names of individual participants.

The second data collection phase occurred at the final scenario-planning workshop for four of the 10 organizations. In the other six organizations, due to timing and scheduling constraints, data were collected two weeks after the conclusion of the scenario-planning workshops and exercises. In these latter cases, participants were asked to attend a meeting specifically to complete the posttest and debrief the project. This was also an opportunity for participants to provide any further feedback on their experiences in the scenario-planning workshops.

Data Analysis. Standard descriptive statistics were generated using IBM SPSS for Macintosh to check the data for a skewness and kurtosis. Means were computed for both pre- and post-test results on each of the seven dimensions of learning organization characteristics. Simple t-tests were performed between pre- and post-test means on each of the seven factors of the DLOQ.

Results. This section presents descriptive statistics and t-tests for the seven research hypotheses. Effect sizes are also provided to clarify research results.

Descriptive Statistics. Descriptive statistics indicated the pattern and shape of the sample distribution supported normal distribution (Hair, Black, Babin, Anderson, \& Tatham, 2006; Leech, Barrett, \& Morgan, 2005). Skewness values ranged from -0.47 to -0.18 and kurtosis values ranged from -0.48 to 0.61 .

Table 3 provides general descriptive statistics.

Reliability. Reliability estimates were calculated for each of seven dimensions of the DLOQ scale scores. For the pretest, Cronbach's alpha for the Continuous Learning items was 0.86 ( 0.79 on posttest). The second set of items (Dialogue and Inquiry) had a Cronbach's alpha of 0.70 ( 0.69 on posttest). Cronbach's alpha for the Collaboration and Team Learning items was 0.70 (0.67 on posttest), pretestposttest and the alpha would not have increased with the deletion of any item. The Embedded Systems items had a Cronbach's alpha of 0.71 ( 0.63 on posttest), and 0.73 ( 0.72 on posttest) for the Empowerment items. System Connections scores were 0.80 on the pretest and 0.67 on the posttest. And finally Leadership items were 0.86 on the pretest and 0.81 for the posttest scores. Cronbach's alpha scores exceeded the acceptability level of 0.7 with the exception of the Collaboration and Team Learning (posttest) items, Embedded Systems posttest items, and System Connections posttest. Overall, the reliability estimates are generally within acceptable ranges, indicating consistent scores were generated since no scores fell below 0.60 .

Paired Samples t-tests. The primary analysis tool for this research study was seven paired sample t-tests, one for each of the seven dimensions of learning organization characteristics.

Assumptions. Nimon (2011) wrote a call to action for increasing the quality of quantitative research studies. In it, she discusses the primary importance 
Table 3. Descriptive Statistics for Participant Responses on the DLOQ.

\begin{tabular}{llllcc}
\hline & & Mean & $N$ & Std. Deviation & Std. Error Mean \\
\hline \multirow{2}{*}{ Pair 1 } & ContLearnPre & 4.00 & 133 & 1.03 & 0.09 \\
& ContLearnPost & 4.09 & 133 & 0.83 & 0.07 \\
\multirow{2}{*}{ Pair 2 } & DiaInqpre & 3.69 & 133 & 0.82 & 0.07 \\
& DiaInqpost & 4.02 & 133 & 0.86 & 0.07 \\
\multirow{2}{*}{ Pair 3 } & TeamLearnPre & 3.78 & 133 & 0.81 & 0.07 \\
& TeamLearnPost & 4.20 & 133 & 0.68 & 0.06 \\
\multirow{2}{*}{ Pair 4 } & EmbedSysPre & 3.41 & 133 & 0.82 & 0.07 \\
\multirow{4}{*}{ Pair 5 } & EmbedSysPost & 3.91 & 133 & 0.71 & 0.06 \\
& EmpowerPre & 3.85 & 133 & 0.90 & 0.08 \\
Pair 6 & EmpowerPost & 4.12 & 133 & 1.33 & 0.12 \\
& SystConnectPre & 3.91 & 133 & 0.91 & 0.08 \\
Pair 7 & SystConnectPost & 4.12 & 133 & 0.82 & 0.07 \\
& LeadershipPre & 3.96 & 133 & 0.99 & 0.09 \\
& LeadershipPost & 4.24 & 133 & 0.86 & 0.07 \\
\hline
\end{tabular}

of addressing the assumptions dictated by the particular statistical test used. The assumptions underlying t-tests are that the data are normally distributed, and that the variation in scores between groups is not reliably different. The normality assumption is shown as met by the descriptive statistics reported above (skewness and kurtosis), and the variation in scores between groups were examined using Hierarchical Linear Modeling (HLM). For the analysis, we computed difference scores from pre- to posttests, and analyzed the data using the interclass correlation coefficient (ICC). The ICC score provides an indication of the amount of variance that is accounted for among a series of groups, taking into account the nestedness of the data. The ICC scores for the seven dimensions were 0.11 , or $11 \%$ for Continuous Learning, 0.04 , or $4 \%$ for Dialogue and Inquiry, 0.04 , or $4 \%$ for Team Learning, 0.01 , or $1 \%$ for Embedded Systems, 0.01, or 1\% for Empowerment, 0.18, or 18\% for System Connections, and finally 0.13 , or $13 \%$ for Leadership. According to Lee (2000) any ICC value exceeding 11\% would require closer examination. Three factors exceeded 11\% (Continuous Learning, Empowerment, and System Connections), and upon closer examination, the grand means from the HLM analysis did not substantially differ from the grand means that resulted from the paired samples t-test. In other words, the analysis indicates that there was insignificant variability among the groups, meeting the assumption that variation in scores across the groups is not reliably different, and lending further evidence to support generalizability of the results. In the 
interest of reporting the results as parsimoniously as possible, the t-test results are presented.

The t-test results indicate scenario planning can be associated with improved perceptions of learning organization characteristics for six of the seven dimensions. For all dimensions except "Continuous Learning," we can therefore reject the null hypothesis that the intervention would have no effect. Table 4 also includes effect sizes as estimates of the magnitudes of the effects within the sample. Approximate guidelines for interpreting Cohen's d are 0.2 to 0.3 for a small effect size, 0.4 to 0.8 for a moderate effect size, and anything over 0.8 could be considered a large effect size (Cohen, 1988). Table 4 shows the results of the seven t-tests.

Results indicate strong changes in perceptions of Team Learning and Embedded Systems, moderate changes in perceptions of Dialogue and Inquiry, System Connection, and Leadership, and small changes in perceptions of Empowerment. Results show insignificant changes in perceptions of Continuous Learning.

\section{Discussion}

The study results show a general increase in participant perceptions of six of the seven learning organization dimensions after participation in scenario planning; however, not all increased equally. Of the six, Dialogue and Inquiry, Team Learning, and Embedded Systems are all dimensions that scenario planning features explicitly as part of its intended impact areas (and those dimensions carried the most improved changes in perceptions). In other words, dialogue is the hallmark of scenario-based approaches strategic problems, and its practical application focuses on these elements explicitly. The results indicate that participants viewed these three categories as improving most positively after the scenario exercise. These three categories seem like obvious outcomes of scenario planning in terms of its relation to learning organizations and our results have confirmed an association.

Leadership, System Connection, and Empowerment also showed improved perceptions over the course of the scenario project as might be expected. These three categories all could be called "by-products" of scenario planning; they are less well understood, less well documented, and less well hypothesized in the scenario-planning literature.

These three categories are less obvious, though logical theoretical explanations can be quickly constructed. Leadership, for example, is a phenomenon that may not have immediate obvious connection to scenario planning, and scenario planning has not been studied or documented as a leadership development tool or intervention. It seems apparent, however, that scenario planning is a tool leaders might find extremely useful in a turbulent business environment, and the capability to facilitate scenario planning could be viewed as a valuable skill. In other words, the ability to negotiate complex 


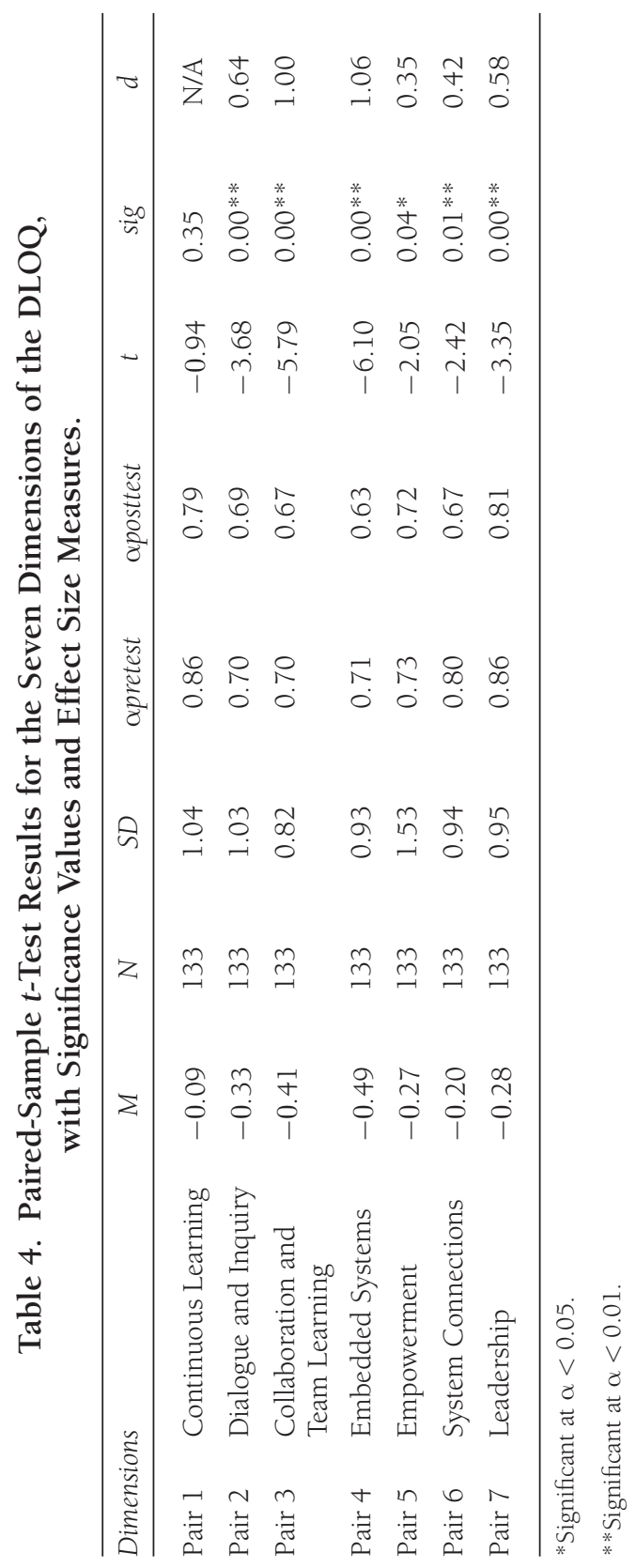


problems, issues, and ideas among a team of decision makers seems like a logical and valuable leadership skill.

System Connection may have shown improved perceptions because scenario planning asks participants to try to understand the systems dynamics of their situation. For example, many scenario exercises involved participants mapping the business model and then "wind tunneling," or testing, it through the scenarios. The purpose is to explore the impacts of the dynamics in the scenarios on human resources, finances, resource allocation strategies, research and development investments, and others. Scenarios tell stories about the external and internal organizational environments and arrange variables in interesting and surprising ways. Each scenario, therefore, can be thought of as a systems diagram (and scenarios are sometimes mapped this way), and a key purpose of scenario planning is to help decision makers understand how their decisions influence the interaction between the organization and its environment.

Empowerment is a dimension that may have improved because of the nature of scenario planning and its departure from typical strategic planning retreats. Scenario planning asks for input from all levels of the organization and creates space for genuine dialogue among those levels of the organization. In other words, scenario planning gets people involved in planning who are typically and traditionally excluded from such an activity. For many line and middle managers, it is the first time they have had an opportunity to provide their perspective to upper management in a format other than a survey. Indeed one goal of scenario planning is to instigate a strategic conversation throughout the organization-it is not reserved for executives only. These characteristics of scenario planning may provide the impetus for further research to understand if and how participants feel empowered to change and prepare their unit for an uncertain future.

Finally, Continuous Learning scores (the only insignificant result on the $t$-test) may not have changed over the intervention because scenario planning is often a "one-time" effort in organizations. Few organizations have fully adopted scenario planning in a continuous planning cycle, and the simple lack of continued use over time may be the underlying reason for insignificant results on this dimension. Logically, understanding how often scenario planning is practiced in varying industries would be helpful in more fully understanding this result, and forms a clear research question for additional study.

Overall, the results show a promising connection between scenario planning and learning organization culture. Of course, continued study will help not only to further establish this connection, but also to affirm the utility of scenario planning beyond a current fad in strategic planning. The results here differ slightly from the previous study using 10 participants. In that study, five of the seven dimensions showed significant improvement in perceptions, and the two that did not show significance differed from the current study. Table 5 
Table 5. Comparisons of Current Significant Results with Previous Significant Results.

\begin{tabular}{lcc}
\hline & Significant Current Study & 2006 Study \\
\hline & No & Yes \\
Dialogue and Inquiry & Yes & Yes \\
Collaboration and Team Learning & Dimensions & Yes \\
Embedded Systems & Continuous Learning & No \\
Empowerment & Yes & No \\
Systems Connections & Yes & Yes \\
Leadership & Yes & Yes \\
\hline
\end{tabular}

displays a comparison of significant results between the current and previous studies.

Explanations for these differing results are not obvious. There are numerous possible intervening factors such as team dynamics, and previous approaches to planning that are not accounted for in either study. It is clear that yet further replication studies are the only way to pin down any potential pattern of scenario-planning impact. However, both studies showed noticeable increases in participant perceptions of numerous dimensions of learning organization characteristics, and this line of research seems worthy of continued inquiry.

Limitations and Recommendations for Future Research. There are several limitations to this research that must be considered in interpreting the results, and naturally provide the opportunity for future research. These limitations and recommendations are described with particular attention to improving scenario-planning research overall. First, some general limitations are described, followed by more particular limitations associated with a onegroup, pre- and post-test design.

General Limitations. Two major limitations to this research are (1) lack of a control group, and (2) potential facilitator inconsistency or bias. The lack of a control group is a serious limitation to the study that prevents the ability to establish causation. While we have found some strong effect sizes, the study does not allow for the ability to predict improved perceptions of learning organization characteristic as an outcome of scenario planning. We have, however, found additional evidence that scenario planning and learning organization characteristics are associated, and understanding more details about the nature of that association is the next logical step. This limitation can be overcome simply by establishing a control group in a future study. While sometimes difficult to establish in organizational research, a 
control group would solidify the ability to make more assertive generalizations about the effects of scenario planning. It could be argued that the diversity captured in this study actually strengthens the findings that scenario planning is effective in a wide variety of settings and situations. Further research can be more intentional in selecting organizations to tease out just what the differences are.

Facilitator bias is one indicator that could influence the effectiveness of the scenario planning exercise (van der Heijden, 2005; Chermack \& van der Mewe, 2003). Because the data in the present study is a compilation of data from 10 scenario-planning workshops conducted by 10 teams, it is not clear how and where facilitation difference may have impacted the effectiveness of the exercise. However, it should be noted that all teams used the general framework in Figure 1 to build their workshop designs and deliver the scenario exercise. Deeper studies on characteristics of facilitators and their interactions with clients are certainly needed and are as yet an undocumented component of scenario planning. Critical reflection is also needed to understand how meaning is created between facilitators and participants, and between participants. Further, the results of the HLM analysis indicate insignificant variation across the groups, meaning that facilitator bias was not an issue in this study as the effects were distributed. While variation of results across the organization was not a focused component of our research question, these results are critically important for scenario-planning researchers and practitioners, as they support the notion that the intervention worked across the organizations regardless of facilitation, industry, size, and many other possible factors.

One-Group, Pre- and Post-Test Design Limitations. There are specific threats to validity associated with a one-group, pre- and post- test research design (Campbell and Stanley, 1963). These are (1) history, (2) testing, and (3) maturation. History refers to some other event that coincided with the treatment that may have influenced or caused the results. Without a control group it is difficult to know that the scenario-planning exercise was the cause of the changes we found in our research results. Other organizational interventions unknown to the researchers may have had an impact; however, the use of a variety of data sources reduces this limitation somewhat. Testing refers to the effects of repeated testing; for example, participants may have recognized items on the instrument, and therefore results may have been affected by recall, or familiarity. Finally,maturation refers to natural changes in participants over time. Individual interests, education, or other workshops may have introduced concepts that affected their responses. We have tried to mitigate this effect by issuing the posttest as soon as possible after the intervention. These three limitations underscore the importance of a control group. Again, while using control groups can be difficult in organizational research, it is clearly worth the effort to ground research results, rule out some critical limitations, and increase the validity of responses. 


\section{Conclusions}

This research study has found evidence for association between scenario planning and participant perceptions of learning organization characteristics. This study was a replication of a previous study that featured only nine participants. The replication improved the design by garnering a total of 133 participants from 10 organizations, lending additional credibility to the results. The lack of a control group prevents us from drawing predictive conclusions. However, the sample size constitutes one of the largest research studies on scenario planning to date, with numerous opportunities to improve and continue studying scenario planning well into the future.

Obvious next steps in the research will be to attempt to overcome the issue of convenience sampling, consider alternative measures of the learning organization, and dig more deeply into the significant results that are less well understood and documented in the scenario literature.

To address the issue of convenience sampling would require some effort. One approach underway is to investigate which of the Fortune 1000 have engaged in scenario planning. High-ranking managers of companies on the Fortune 1000 list who had previously engaged in scenario planning could be sent the DLOQ and assessment could be made on a larger scale regarding the link between scenario planning and perceptions of learning organization characteristics. However, numerous limitations are introduced, such as potential differing approaches to scenarios among the organizations, the recentness and consistency of the scenario effort, and using just a single perception data point in each organization. However, using publicly traded companies would yield the possibility of replicating a portion of Ellinger, Ellinger, Yang, and Howton's (2003) research that linked the DLOQ results to financial performance.

Alternative approaches to measuring learning organization characteristics are also needed. Theories, frameworks, and instruments exist by authors other than Watkins and Marsick, (e.g., Garvin et al, 2008; Hannah and Lester, 2009), and it would be a clear advantage to bring in a variety of these measures of learning organization characteristics. Additional measures would enrich any positive results we might find.

While the Leadership, System Connections, and Empowerment dimensions showed significant perceptual increases, it is less clear why these dimensions would improve. General research into the link between leadership and scenario planning would be useful. McWhorter, Lynham, and Porter have begun this line of research and continued effort in this area will aid in understanding how these two phenomenon interact more completely. System Connections seems like it may be an obvious connection given the utility of system theory in scenario planning; however, there may be more specific relationships that require study. For example, Watkins and Marsick described System Connections as also 
including how employees might see their contribution to the organization. Thus, engagement and job satisfaction might be useful additional areas to explore. Finally, Empowerment is not a well-understood variable in the context of scenario planning. The concept does not arise in scenario literature and could be a variable missed by Chermack's theorizing $(2004,2005)$.

Future research is clearly needed that makes use of control groups, targets facilitator characteristics, and addresses some of the less clearly understood relationships uncovered in this study (e.g., the link between scenario planning and leadership, systems connection, and empowerment). Furthermore, because not all of the dimensions that increased did so at the same rate, another avenue for future research would be to test if this pattern holds across studies. These are all logical places where scenario-planning research can contribute to the emerging and growing body of evidence for scenario-planning outcomes. Logical research questions are associational, such as, "What is the relationship between scenario planning and leadership?," "Does scenario-planning make systems thinkers?," "Do scenarioplanning participants feel more empowered to influence the courses of their organizations?," and "What are characteristics of effective scenario-planning facilitators?" Any of these are good places to make progress in generating new knowledge about scenario planning.

In conclusion, our study has contributed valuable data points to the scenario and learning organization literatures. Also, we have taken another step in using data to denote scenario planning as HRD's strategic learning tool (Chermack $\&$ Swanson, 2008) with the significant results relating scenario planning to increased perceptions of learning organization characteristics.

\section{References}

Boje, D. M. (1991). The storytelling organization: A study of storytelling performance in an office supply firm. Administrative Science Quarterly, 36, 106-126.

Bradfield, R. (2008). Cognitive barriers in the scenario development process. Advances in Developing Human Resources, 10(2), 198.

Bradfield, R. Wright, G., Burt, G., Cairns, G., \& van der Heijden, K. (2005). The origins and evolution of scenario techniques in long range business planning. Futures, 37(8), 795-812.

Campbell, D., \& Stanley, J. (1963). Experimental and quasi-experimental designs for research. Boston, MA: Houghton Mifflin.

Chermack, T. J. (2003). A methodology for assessing performance-based scenario planning. Journal of Leadership and Organization Studies, 10(2), 55-64.

Chermack, T. J. (2011). Scenario planning in organizations: How to create, use and assess scenarios. San Francisco, CA: Barrett-Koehler.

Chermack, T. J., Lynham, S. A., \& van der Merwe, L. (2006). Exploring the relationship between scenario planning and perceptions of learning organization characteristics. Futures, 38(7), 767-777.

Chermack, T J., \& Swanson, R. (2008). Scenario planning: Human resource development's strategic learning tool. Advances in Developing Human Resources, 10(2), 129. 
Chermack, T., \& van der Merwe, L. (2003). The role of constructivist learning in scenario planning. Futures, 35(5), 445-460.

Chermack, T. J., van der Merwe, L., \& Lynham, S. A. (2007). Exploring the relationship between scenario planning and strategic conversation quality. Technological Forecasting and Social Change, 74(3), 379-390.

Cohen, J. (1988). Statistical power analysis for the behavioral sciences (2nd ed.). Lawrence Erlbaum Associates.

De Geus, A. P. (1988). "Planning as learning." Harvard business review, 66(2), 70.

Durance, P., \& Godet, M. (2010). Scenario building: Uses and abuses. Technological Forecasting and Social Change, 77(9), 1488-1492.

Ellinger, A., Ellinger, A., Yang, B., \& Howton, S. (2003). "Making the Business Case for the Learning Organization Concept. Advances in Developing Human Resources 5(2), 163-172.

Garvin, D., Edmondson, A., \& Gino, F., (2008). "Is yours a learning organization?," Harvard Business Review, 86(3), 109-116.

Hair, J. F., Black, W. C., Babin, B. J., Anderson, R. E., \& Tatham, R. L. (2006). Multivariate data analysis (6th ed.). Englewood Cliffs, NJ: Prentice-Hall.

Hannah, S. T., \& Lester, P. (2009)."A multilevel approach to building and leading learning organizations." The leadership quarterly, 20(1), 34.

Kildruff, M., \& Mehra, A. (1997). Post-modernization and organizational research. The Academy of Management Review, 22(2), 453-481.

Leech, N. L., Barrett, K. C., \& Morgan G. A. (2005). SPSS for intermediate statistics: Use and interpretation. Mahwah, NJ: Lawrence Erlbaum Associates.

Marsick, V. J., \& Watkins, Karen E. (1999). Facilitating learning organizations: Making learning count. Brookfield, VT: Gower.

Marsick, V., \& Watkins, K. (2003). Demonstrating the value of an organization's learning culture: The dimensions of the learning organization questionnaire. Advances in Developing Human Resources, 5(2), 132-151.

Maruyama, M. (2004) Peripheral vision: polyocular vision or sub-understanding. Organization Studies, 25(3), 467-480.

Michael, D. N. (1995). Barriers and bridges to learning in a turbulent human economy. In L. Gunderson, C. Holling \& S. Light (Eds.), Barriers and bridges to the renewal of ecosystems and institutions, (461-485). New York, NY: Columbia University Press.

Mintzberg, H., Ahlstrand, B. W., \& Lampel, J. (2005). Strategy safari: A guided tour through the wilds of strategic management. New York, NY: Simon \& Schuester.

Nimon, K. (2011). Improving the quality of quantitative research reports: A call for action. Human resource development quarterly. 22(4), 387.

Pink, D. (2005). A story goes with it. Forbes, 175(4), 30.

Pink, D. (2006). A whole new mind: Why right brainers will rule the future. New York, NY: Riverhead Books.

Porter, M. E. (1985). Competitive advantage. New York, NY: Free Press.

Rowden, R., \& Conine, C., (2005) The impact of workplace learning on job satisfaction in small US commercial banks. Journal of Workplace Learning, 17(4), 215-230.

Schwartz, P. (1996). The art of the long view: Planning for the future in an uncertain world. New York, NY: Doubleday.

Shaw, G., Brown, R., \& Bromily, P. (1998) Strategic Stories: How 3M is Rewriting Business Planning, Harvard Business Review, 76(3) 41-50.

Song, J. H., \& Chermack, T. (2008) A theoretical approach to the organizational knowledge formation process: Integrating the concepts of individual learning and learning organization culture. Human Resource Development Review, 7(4), 424-442.

Song, J. H., Joo, B. K., \& Chermack, T. J. (2009). The dimensions of learning organization questionnaire (DLOQ): A validation study in a Korean context. Human Resource Development Quarterly, 20(1), 43-64. doi: 10.1002/hrdq.20007 
Thomas, C. W. (1994). Learning from imagining the years ahead. Planning Review, 22(3), 6-10. Tucker, K. (1999). Scenario planning. Association Management, 51(4), 70-75.

van der Heijden, K. (1997). Scenarios: The art of strategic conversation. New York, NY: John Wiley. van der Heijden, K. (2005). Scenarios: The art of strategic conversation (2nd edition). England: John Wiley \& Sons.

Wack, P. (1985a). Scenarios: Shooting the rapids. Harvard Business Review, 63(6), 139-150.

Wack, P. (1985b). Scenarios: Uncharted waters ahead. Harvard Business Review, 63(5), 73- 89.

Wack, P. (1985c). Scenarios: The gentle art of re-perceiving. Unpublished manuscript, Cambridge, MA: Harvard Business School.

Watkins, K. E., and Marsick, V. J. (1996). Adult educators and the challenge of the learning organization, Adult Learning, 7(4), 18-20.

Watkins, K. E., and Marsick, V. J. (1997). Dimensions of the learning organization questionnaire [Survey]. Partners for the learning organization. Warwick, RI.

Watkins, K. E., and Marsick, V. J. (2003). Demonstrating the value of an organization's learning culture: the dimensions of the learning organization questionnaire. Advances in developing human resources, 5(2), 132-151.

Wright, G., \& Goodwin, P. (1999). Future-focused thinking: Combining scenario planning with decision analysis. Journal of Multi-Crit+eria Decision Analysis, 8, 311-321.

Wright, G., van der Heijden, K., Burt, G., Bradfield, R., \& Cairns, G. (2008). Scenario planning interventions in organizations: An analysis of the causes of the success and failure. Futures, 40(3), 218.

Yang, B. (2003). Identifying valid and reliable measures for dimensions of a learning culture. Advances in Developing Human Resources, 5(2), 152.

Yang, B., Watkins, K. E., \& Marsick, V. J. (2004). The construct of the learning organization: Dimensions, measurement, and validation. Human Resource Development Quarterly, 15(1), 31-55.

Melissa Haeffner is with the Graduate Department Program of Ecology, Human Environment Interactions, Colorado State University, Fort Collins, Colorado.

Deanna Leone is with the Division of Student Affairs, Colorado State University, Fort Collins, Colorado.

Laura Coons is with Colorado State University, Fort Collins, Colorado.

Thomas Chermack is with the Scenario Planning Institute, Colorado State University, Fort Collins, Colorado. 